\title{
The effect of sperm preparation medium on malondialdehyde concentration in the seminal plasma of normozoospermic and oligoastheno- teratozoospermic patient
}

\author{
تأثير إضافة وسط تنشيط النطف إلى البلازما المنوية على تركيز المالوندايألايهايد

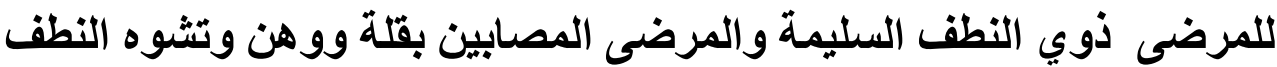

\section{Maha Khalid Abd-Al Kareem}

Dept. of Clinical Rreproductive Physiology/ Institute of Embryo Researches and Infertility Treatment/ Al-Nahrain University

$$
\begin{aligned}
& \text { مها خالد عبد الكريم } \\
& \text { قسم فسلجة التناسل السريري/ معهد أبحاث الأجنة وعلاج العقم/جامعة النهرين }
\end{aligned}
$$

\begin{abstract}
Intra uterine insemination (IUI) has been widely used to treat infertility. Infertility may be due to increased levels of reactive oxygen species (ROS) in the seminal plasma. The goal of our study was to confirm that using sperm preparation medium to activate the spermatozoa in IUI might be helpful to decrease the levels of ROS in the seminal plasma, lowering the oxidative stress and the damage effects of the sperm and enhance a good quality sperm capable of capacitation, acrosomal reaction and fertilization. The levels of malondialdehyde (MDA) were measured in the seminal plasma in 40 infertile men of normozoospermia and oligoasthenoteratozoospermia before and after adding medium to the samples. Results showed that there was a significant $(\mathbf{P}<0.05)$ decrease in all parameters of semen samples in oligoastheno-teratozoosperia as compared with the other group, there was no significant increase in the level of MDA before adding the medium to the samples and a significant $(P<0.05)$ increase after adding the medium to the same samples in the second group as compared with normozoospermic patients. Results reported in this study, clearly indicated that adding sperm preparation medium to the semen samples would improve the fertilization rates by decreasing the oxidative damages to the spermatozoa. Our results might be need a further studies to find a clue for the treatment of infertility in man.
\end{abstract}

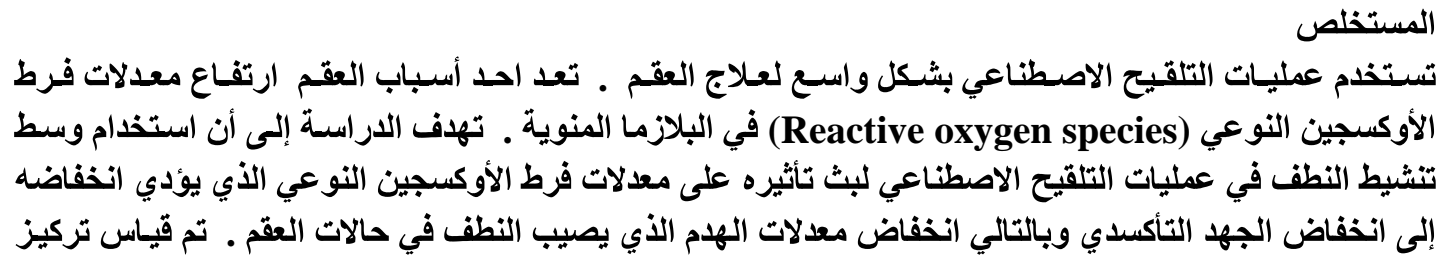




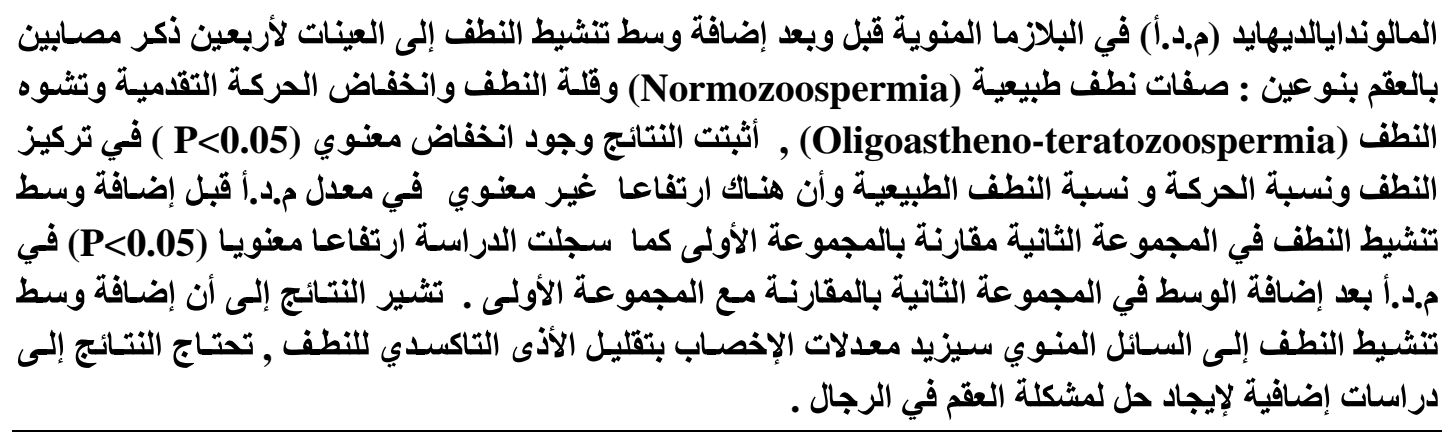

\section{Introduction:}

Reactive oxygen species (ROS) are free radicals that play a significant role in many of the sperm physiological processes such as capacitation, hyperactivation, and sperm-oocyte fusion [1]. However, they also trigger many pathological processes in the male reproductive system, and these processes have been implicated in cancers of the prostate, as well as in male infertility [2]. There are two main sources of ROS in semen: leucocytes (neutrophils and macrophages) and immature spermatozoa which lead to sperm dysfunction. A spermatozoon produce ROS mainly when a defect occurs during spermiogenesis that results in retention of cytoplasmic droplets. When levels of ROS overwhelm body's antioxidant defense system, oxidative stress (OS) occurs. OS is a condition in which the elevated levels of ROS damage cells, tissues, or organs [3]. Spermatozoa contain large quantities of polyunsaturated fatty acids (PUFA) and therefore they are susceptible to ROS-induced damage. It has been suggested that ROS induce membrane lipid peroxidation in sperm and that the toxicity of generated fatty acid peroxides are important causes of sperm malfunction [4].

The most widely used assay for lipid peroxidation involves the measurement of malondialdehyde (MDA)- thiobarbituric acid (TBA) adducts due to its simplicity [5], MDA is an organic compound with the formula $\mathrm{CH} 2(\mathrm{CHO}) 2 . \mathrm{ROS}$ degrade polyunsaturated lipids, forming MDA. This compound is a reactive aldehyde and is one of the many reactive electrophile species that cause toxic stress in cells and form advanced glycation end products. The production of this aldehyde is used as a biomarker to measure the level of OS in an organism.MDA reacts with deoxyadenosine and deoxyguanosine in DNA, forming DNA adducts, primarily M,G, which is mutagenic [6].

This study aimed to assess the effects of sperm preparation medium as antioxidant on some biochemical parameters that include Malondialdehyde (MDA) concentration in the seminal plasma in two types of infertile men :normozoospermic and oligoasthenoteratozoospermic which underwent intra uterine insemination(IUI).

\section{Materials and methods:}

Forty semen samples (20 normal sperm parameters:normozoospermia and 20 abnormal sperm parameters: oligoastheno-teratozoospermia) from patients conducted to Institute of Embryo Researches and Infertility Treatment / Al- Nahrain University were obtained by masturbation method after sexual abstinence period of 2-3 days. After liquefaction samples were analyzed according to World Health Organization (WHO) guidelines, [7]. Sperm parameters were considered normal when sperm concentration was $\geq 20 \times 10^{6} / \mathrm{ml}$ of semen, motility $\% \geq 50 \%$, normal sperm forms $\% \geq 30 \%$ and leucocytes $\leq 1 \times 10^{6} / \mathrm{ml}$ of 
semen according to (WHO). Semen was prepared using two different methods to separate sperm from seminal plasma [8]:

1. Centrifugation method: This method includes separation of the good motile sperm by allowing them to" swim up"into a layer of sterile culture medium. The procedure involves adding sterile culture medium over liquefied semen, centrifuged at $2500 \mathrm{rpm} / 10 \mathrm{~min}$. Remove the supernatant and the pellet was resuspended in a clean sterile medium ( sperm wash) for $25 \mathrm{~min}$. then isolate the upper layer for IUI .

2. Simple layer technique: This method includes layering sterile culture medium over liquified semen for $20 \mathrm{~min}$. Then isolate the upper layer for IUI.

The presence of $>10 \times 10^{6} / \mathrm{ml}$ of normal motile sperm after preparation seem to provide a realistic cut off, below which pregnancy rates plummet.

Seminal plasma collection: After sperm preparation the seminal plasma were taken after centrifugation for $10 \mathrm{~min}$. at 5000rpm and divided it into two portions one directly refrigerated after transferred into appendorf tubes at $(-4 \mathrm{C})$ and the other portion was mixed with the same amount of sperm preparation culture medium, centrifuged for 10min. at 5000rpm, then isolate the top layer which contained the seminal plasma and the medium into appendorf tubes, refrigerated at $(-4 \mathrm{C})$.

MDA measurement: Lipid peroxidation is based on the reaction of malondialdehyde (MDA) with thiobarbituric acid (TBA); forming a MDA-TBA2 adducts that absorbed strongly at $532 \mathrm{~nm}$, This reaction is the most popular method for estimating MDA in biological samples [9]. MDA in the seminal plasma was determined by the TBA assay [10]. Reagent was prepared by mixing $0.67 \mathrm{gm}$ of 2-thiobarbituric acid (TBA) dissolved in $100 \mathrm{ml}$ of distalled water (D.W) with $0.5 \mathrm{gm}$ of solid sodium hydroxide $(\mathrm{NaOH})$ then 100 $\mathrm{ml}$ of glacial acetic acid was added. Add 0.5 of this reagent to $0.1 \mathrm{ml}$ of seminal plasma and $0.9 \mathrm{ml}$ D.W.. The samples were heated in a boiling water bath for 45 minutes, cooled and centrifuged to 10 minute at $(4000 \mathrm{rpm})$ and the absorbance of the supernatent was read on a spectophotometer at wavelength of 534nm.MDA level were analyzed using method of Rao et al. 1989.

Seminal plasma MDA-TBA concentration $=\frac{\mathrm{Ab} \times 10^{6} \times 10^{3} \times \mathrm{Vs}}{\left(1.56 \times 10^{5}\right) \mathrm{X} \mathrm{Vt}}$

Where:Ab=absorbance of the sample; $\mathrm{Vs}=$ Sample volume; $\mathrm{Vt}=$ total volume and $1.56 \mathrm{X} 10^{\wedge} 5=$ molar absrption coeffecient for MDA-TBA complex [11].

Statistical analysis: Values reported are means \pm SEM. All data were normally distributed and underwent equal variance testing. Statistical significance of difference was determined by SPSS program at 11.5 versions for windows. Average comparsion between two groups was made using Student's $t$-test. $\mathrm{P}<0.05$ was considered as statitistically significant.

\section{Results:}

The sperm concentration $(\mathrm{m} / \mathrm{ml})$, sperm motility\%, sperm normality\%, and the concentration of MDA in the seminal plasma of 20 infertile men with normozoosperia and 20 infertile men with oligoasrheno-teratozoospermia prepared for IUI before and after 
adding sperm preparation medium were determined and compared the results between the two groups.

Table (1) showed the results of semen analysis which include sperm concentration $(\mathrm{m} / \mathrm{ml})$, sperm motility\% and sperm abnormal morphology\% in both groups, all these parameters recorded a significant difference $(\mathrm{P}<0.05)$ between the two groups.

Table (1): Sperm quality parameters of normozoospermia and oligoteratozoospermia in infertile Men

\begin{tabular}{|l|l|l|l|}
\hline Subjects & $\begin{array}{l}\text { Sperm } \\
\text { concentration } \\
(\mathrm{m} / \mathrm{ml})\end{array}$ & Sperm motility\% & $\begin{array}{l}\text { Sperm normal } \\
\text { morphology\% }\end{array}$ \\
\hline Normozoospermia $\mathrm{n}=20$ & $47.16 \pm 3.29$ & $56.61 \pm 4.40$ & $43.88 \pm 3.96$ \\
\hline $\begin{array}{l}\text { Oligoastheno- } \\
\text { teratozoospermia } \mathrm{n}=20\end{array}$ & $12.88 \pm 2.54^{*}$ & $20.16 \pm 4.54^{*}$ & $18.94 \pm 3.70^{*}$ \\
\hline P value & 0.014 & 0.045 & 0.039 \\
\hline
\end{tabular}

Not: The values are the means \pm SEM; $n=$ number of subjects; $* P<0.05$

MDA concentration in the seminal plasma was shown in table 2, there was no significant increase in MDA concentration in patients of oligoastheno-teratozoospermic group compared with the normal group, and there was a significant $(\mathrm{P}<0.05)$ increase in the level of MDA of the second group after adding the medium to the samples as compared with the normal group.

Table (2): seminal plasma MDA concentration before and after adding sperm preparation medium of normozoospermia and oligoteratozoospermia in infertile men

\begin{tabular}{|c|c|c|}
\hline Subjects & $\begin{array}{c}\text { MDA-before } \\
(\mathrm{nmol} / \mathrm{ml})\end{array}$ & $\begin{array}{c}\text { MDA-after } \\
(\mathrm{nmol} / \mathrm{ml})\end{array}$ \\
\hline Normozoospermia $\mathrm{n}=20$ & $168 . .126 \pm 10.06$ & $93.68 \pm 9.67$ \\
\hline Oligoastheno-teratozoospermia $\mathrm{n}=20$ & $322.71 \pm 12.29$ & $124.04 \pm 12.00^{*}$ \\
\hline P value & 0.24 & 0.049 \\
\hline
\end{tabular}

Not: The values are the means \pm SEM; $n=$ number of subjects; $* \mathbf{P}<0.05$

\section{Discussion:}

In recent years, successful fertility results after artificial insemination (AI),[12]. Sperm preparation medium used to activate sperm which prepared for assisted reproductive techniques (ART) including AI. This medium contains materials like: Glucose, sodium pyruvate, sodium bicarbonate and penicillin-streptomycin, it besides making wash to the sperm, it also helps with these contents lowering the OS in the seminal plasma which affect on the sperm activity due to its antioxidant, antiapoptotic effects and scavenging $\mathrm{NO}^{*}$, regulation of pathological conditions caused by excessive generation of $\mathrm{NO}^{\circ}$ and its oxidation product, peroxynitrite and then improvement of fertilization rates [13]. In addition, the intracellular antioxidant enzymes cannot protect the plasma membrane that surrounds the acrosome and the tail, forcing the spermatozoa to supplement their limited intrinisc antioxidant defence by depending on the protection afford by the seminal plasma [14]. Our results found that the content of MDA in the seminal plasma is much higher in 
oligoteratotozoospermia before treated the samples with sperm preparation medium when compared with the other samples, because MDA is a by-product of oxidative stress, elevation of its level indirectly reflects high levels of ROS in the seminal plasma [15]. Oligoasthenozoosperm is associated with higher seminal MDA activity. It was suggested that the lipid peroxidation, which represented by the MDA activity, might compromise the sperm viability. Increased MDA activity could represent the pathologic lipid peroxidation of spermatozoa membrane and the following inhibition of sperm motility and viability [16].

Oxidative damage is common for spermatozoa during epididymal maturation and storage [17]. Human spermatozoa are highly susceptible to oxidative injury but are naturally protected from such injury by the antioxidant properties of the seminal plasma. ROS plays a central role for sperm physiology such as sperm maturation and capacitation. Abnormal ROS production is associated with defective sperm function. A fine balance between ROS production and recycling is essential for spermatogenesis. Among the various methods for detection of lipid peroxidation, we choose to measure spontaneous MDA production, which reflects the peroxidation of polyunsaturated phospholipids, the major components of sperm membrane. MDA measurements are physiological and relevant because major loss of sperm function may occur with minimal damage to the membranes that envelop the sperm and/or divide key intracellular sperm compartments. The high MDA production in the male is associated with low fertilization rates in the in vitro fertilization (IVF) process [13]. The reduction of MDA by using antioxidant therapy was correlated with the improvement of fertilization rates. Real role of MDA and other antioxidants upon the sperm qualities merits further surveys. In vitro supplements used during sperm preparation and assisted reproductive techniques also help to protect the spermatozoa against ROS. Moreover, adding antioxidants to the culture media neutralizes ROS produced by the leukocytes and immature spermatozoa and improves sperm-oocyte fusion [18]. Sperm preparation medium has a beneficial effect on sperm motility and acrosomal reaction and reduces the $\mathrm{O}_{2}^{--}$released by the human spermatozoa, protects the spermatozoa against oxidative damage induced by $\mathrm{H} 2 \mathrm{O} 2$ [19].

Conclusions : Spermatozoa are under a continuous influence of OS because of excessive generation of ROS. Although spermatozoa are affected in different ways by OS, there was sufficient antioxidant protections that can decrease the progression of the damage. However, when an imbalance exists between levels of ROS and the natural antioxidant defenses, various measures can be used to protect spermatozoa against the OS-induced injury. Nevertheless, further studies are required to validate their use in these groups of patients. Roles of seminal MDA upon spermatogenesis merits further surveys

\section{References:}

1. Aitken, RJ (1994). A free radical theory of male infertility. Reprod.Fertil.Dev., 6: 1923.

2. Agarwal, A and Said, T (2005). Oxidative stress,DNA damage and apoptosis in male infertility : a clinical approach.BJU International.,95: 506-507.

3. Saleh, R; Agarwal, A; Essam, A et al.,( 2003). Negative effects of increased sperm DNA damage in relation to seminal oxidative stress in men with idiopathic and male factor infertility. Fertil.Steril., (suppl 3): 1597-1605. 
4. Agarwal, A; Prabakaran, S and Allamaneni,A (2006). What an andrologist should know about free radicals and why?. Urology. 67:2-8.

5. Nosratollah, Zarghami and Ali, Khosrowbeygi (2005). Seminal plasma levels of 15F2 $\alpha$-Isoprostane, Malondialdehyde and total Homocysteine in Normo- zoospermic and Asthenospermic Males .Indian J. of clinical biochemistry. 20(2): 86-91.

6. Fraga, CG; Motchnik, PA; Wyrobek, AJ et al., (1996). Smoking and low antioxidant levels increase oxidative damage to sperm DNA .Mutat.Res., 351: 199-203.

7. World Health Organization (1999): Laboratory manual for examination of human semen and semen cervical mucus interaction, $4^{\text {rd }}$ ed.,Cambridge ,UK :The press syndicate of the University of Cambridge .

8. Shang, X; Keli, Z; Gangchen, Y and Huang, Y(2004). Analysis of lipid peroxidation levels in seminal plasma of infertile men by high -performance liquid chromatography. 50: 411-16

9. http://Www.nwlifescience.com/products/assaykit/tba.mdaanalysis.htm.Analytica methods for Resolving Data from TBA 2 -MDA Reaction Mixtures.

10. Rao, B; Souflir, JC; Martin, M and David, G (1989). Lipid peroxidatin in human spermatozoa as related to midpiece abnormalities and motility. Gamete Res., 24: 12734.

11. Salim, D (2008). Effect of Vitamin E and Zinc Treatment on Oxidative Stress in Some Iraqi infertile males. M.Sc thesis- Institute of Embryo Researches and Infertility treatment, Al-Nahrain University.

12. Jordi, R; Maria, J and Rodrigues, M et al(2005). Survival and in vitro fertility of boar spermatozoa frozen in the presence of superoxide dismutase and or catalase. J. andro

13. Kobayashi, T; Miyazaki, T; Natori, M and Nozawa, S (1991). Protective role of super oxide dismutase in human sperm motility: superoxide dismutase activity and lipid peroxide in human seminal plasma and spermatozoa. Hum Reprod.,6: 987-91.

14. Iwasaki, A and Gagnon, C (1992). Formation of reactive oxygen species in spermatozoa of infertile patients. Fertil.Steril., 57: 409-16.

15. Yao-Yuan, Hsieh; Chi-Chen, Chang and Chich-Sheng, Lin (2006). Seminal malondialdehyde concentration but not glutathione peroxidase activity is negatively correlated with seminal concentration and motility. Int J Biol Sci., 2(1): 23-29.

16. Donelly, E; McClure, N and Lewis, S(1999). Antioxidant supplementation in vitro does not improve human sperm motility. Fertil Steril.,72: 484-495

17. Agarwal,A; nallela,K; Allamaneni, S and Said, T (2004). Role of antioxidants in treatment of male infertility: an overview of the literature.Reprod.Biomed.Online., 8: 616-627.

18. Pedro, M; Fulgencio, P; and Mar?a, I (2007). Sperm lipid peroxidation and proinflammatory cytokines.Asian J Androl; 9 (1): 102-107.

19. Irvin, DS (1996). Glutathione as a treatment of male infertility. Rev Reprod.,1: 6- 12. 